\title{
Characterization of Oligomers by Liquid Ionization Mass Spectrometry. Photopolymerization Process of $p$-Phenylene Diacrylic Acid Diethyl Ester Crystal
}

\author{
Yaming Li*, Takeo Kaneko*, Yoshiyuki Mochida**, Shigeo NaKamURA** \\ and Masahiko TsuchiYA*† \\ *Department of Physical Chemistry, Faculty of Engineering, Yokohama National University, \\ Tokiwadai, Yokohama 240, Japan \\ **Department of Applied Chemistry, Faculty of Engineering, Kanagawa University, \\ Rokkakubashi, Yokohama 221, Japan
}

\begin{abstract}
The photoproducts obtained from $p$-phenylene diacrylic acid diethyl ester crystal with various photo-irradiation times were analyzed by liquid ionization (LI) mass spectrometry. It was found that hexadecane was an effective matrix for observing large oligomer ions, trifluoroacetic acid enhanced the protonated molecular peaks, and 2-aminoethanol was useful to produce stable adduct ions of oligomers. The molecular weight distributions were readily determined from the mass numbers of both protonated molecules and molecular adduct ions. Large oligomers up to the hexamer (M.W. 1644) were observed, even though they were insoluble in $\mathrm{CHCl}_{3}$. The topochemical photopolymerization via step-growth process was observed, as has been reported. The mass spectra of the oligomers produced with longer than 20 min photo-irradiation, however, indicated that the reaction between two dimer molecules occurred also to result in formation of species having an even-numbered degree of polymerization, such as tetramer, hexamer and so on. The observed chain-growth may be attributed to the gradual change of molecular arrangement in the reacting crystal during the initial stage of topochemical reaction. The LI method gives detailed information about chemical reactions.
\end{abstract}

Keywords Liquid ionization mass spectrometry, matrix, oligomer, $p$-phenylene diacrylic acid diethyl ester, mass spectrometry

It has been reported that a series of $p$-phenylene diacrylic acid diesters were polymerized on photo-irradiation via a step-growth mechanism by repeating [2+2] photo-cyclodimerization in the crystalline state. ${ }^{1-3}$ The photopolymerizations of diolefin crystals have been studied extensively by Hasegawa and his colleagues ${ }^{1,2}$, but only a few papers have described the features of chain growth along with the reaction process. ${ }^{4-7}$ The determination of molecular weight distribution of the oligomers in the initial stage may be indispensable for the understanding of the reaction mechanism in the crystalline state.

Liquid chromatography such as size exclusion chromatography is usually used for the determination of molecular weight distribution of oligomers. ${ }^{8}$ However, this method does not give any information on the molecular weight of each fraction and sometimes its resolution is not satisfactory. On the other hand, mass spectrometry is most suitable for the above purpose, because the resolution is very high and the molecular weights of each fraction can be read out directly. ${ }^{9}$

For mass spectrometric characterization of oligomers,

\footnotetext{
† To whom correspondence should be addressed.
}

a variety of soft ionization techniques are commonly applied, such as field desorption (FD) ${ }^{10,11}$, fast atom bombardment (FAB) ${ }^{12}$, electrospray ionization (ESI) ${ }^{13,14}$, and matrix assisted laser desorption ionization (MALDI). ${ }^{15,16}$

Of interest in the present work is the application of liquid ionization (LI) mass spectrometry ${ }^{17-20}$ for the characterization of less- and non-volatile oligomers of $p$-phenylene diacrylic acid diethyl ester (abbreviated as PDAEt). The sequence of reactions is described in Scheme 1. IR and NMR spectroscopies have been generally used to analyze the structures of these photoproducts and the reaction mechanisms. ${ }^{2}$ As to the molecular weight distributions, previous papers ${ }^{2,5-7}$ have reported that some of unsymmetrically substituted diolefin crystals polymerized with preferential formation of the chemical species having an even-numbered degree of polymerization, such as dimer, tetramer, hexamer, octamer and so on. The observed even-numbered degree of polymerization mechanism of these crystals was unambiguously explained and correlated to their crystal structures.

Liquid ionization (LI) is a kind of soft ionization technique, in which excited argon atoms are utilized and a sample is ionized under atmospheric pressure. LI has 


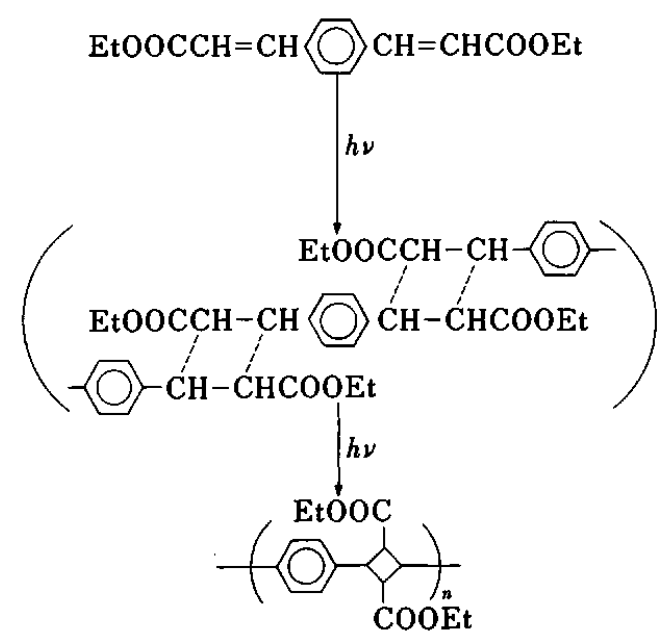

Scheme 1.

several advantages. (1) A matrix having small proton affinity like alkane can be used to enhance protonated molecules for less volatile and thermolabile compounds, such as organometallic compounds, without any fragmentation. ${ }^{20}$ (2) Several reagents possessing polar functional groups such as - $\mathrm{OH},-\mathrm{SH}$, and $-\mathrm{NH}_{2}$, can be used to produce molecular adduct ions. 2-Aminoethanol has been applied to distinguish the isomers of unsymmetrical diolefin dimer ${ }^{21}$ and to determine the molecular weights of methyl hydroperoxyoleates. ${ }^{22}$ (3) Since each component has its own optimum desorption temperature, a mixture can be separated to some extent by programming the sample temperature. Examples have been reported for the separation and determination of polycyclic aromatic hydrocarbons $(\mathrm{PAH})^{23}$ and various compounds. ${ }^{17}$

In the present paper, the application of LI to oligomers derived from the PDAEt crystal with various photoirradiation times is described. Several matrices were examined to enhance the abundance of protonated molecules and/or of adduct ions. The results obtained by LI are compared with their FAB mass spectra. The effects of irradiation times on the molecular weight distribution of oligomers are discussed.

\section{Experimental}

\section{Samples and reagents}

Oligomer crystals of $p$-phenylene diacrylic acid diethyl ester (PDAEt) used in this study were synthesized by photo-irradiation using an USHIO UM-102 super-highpressure mercury lamp $(100 \mathrm{~W})$. The degree of polymerization depends on the irradiation time. The photopolymerization products were obtained at various irradiation times: i.e. $10,20,30,40$ and $60 \mathrm{~min}$, respectively.

Chloroform (Special grade, Junsei Chemical Co. Ltd.), trifluoroacetic acid, ethanol and 2-aminoethanol (Special grade, Wako Pure Chemical Industries Ltd.), and hexadecane (Special grade, Tokyo Chemical Industries Ltd.) were used as purchased.

\section{Instrument}

LI mass spectra were measured using a quadrupole mass spectrometer (Extrel EXM2000) equipped with the LI ion source. The principle of $\mathrm{LI}$ and its operating procedures have been reported in detail previously ${ }^{17,18}$ and only the present study is described here. The applied voltage to the needle (sample holder) is $1.35 \mathrm{kV}$, the distance between the needle tip and pinhole is about $2.5-3 \mathrm{~mm}$, flow rate of excited argon is $1.21 / \mathrm{min}$. For normal measurement (soft setting), the voltages applied to the pinhole, skimmer-1 and skimmer- 2 are 40,20 , and $2.5 \mathrm{~V}$, respectively. A personal computer (NEC 9801BX) was used as the data system. Each mass spectrum was obtained between mass range $100-1250$ $\mathrm{Da}$ or $250-1750 \mathrm{Da}$ in $2 \mathrm{~s} / \mathrm{scan}$. Mass spectra presented in this paper are all the averages for more than 30 mass spectra recorded in succession.

FAB mass spectra were measured by a JOEL JMS AX-500 mass spectrometer, under operating condition of $6 \mathrm{keV}$ xenon atoms bombardment. Glycerol and 2nitrobenzyl alcohol (NBA) were used as a matrix.

Infrared spectra were measured by a JASCO IR- 810 infrared spectrophotometer.

\section{Sample preparation}

About $0.5-2 \mathrm{mg}$ oligomer sample was mixed with $100 \mu$ l hexadecane- $\mathrm{CHCl}_{3}$ solution $(1: 1$, by vol.). The sample produced by $10 \mathrm{~min}$ photo-irradiation of PDAEt crystal was almost dissolved in this matrix solution, while those produced with photo-irradiation time longer than $30 \mathrm{~min}$ were partially dissolved and the sample solutions were not transparent. When trifluoroacetic acid, in which all oligomers were dissolved, was used as a solvent, a sample solution mixed with the matrix solution was used, although it formed an emulsion because hexadecane was insoluble in trifluoroacetic acid. About $0.5 \mu 1$ sample solution was deposited on the needle tip, which was then inserted into the LI ion source. A reagent, 2-aminoethanol diluted with ethanol in the ratio of $1: 5$ by volume was introduced into the ion source through vapor phase. Such reagent is called vapor matrix. The device used in these measurements for introducing vapor matrix was similar to that reported previously..$^{17,18}$ LI mass spectra were recorded repeatedly while the sample temperature was raised from $25^{\circ} \mathrm{C}$ to $250^{\circ} \mathrm{C}$ by means of increasing the voltage of a heater around the sample holder.

\section{Results and Discussion}

Photoproducts of PDAEt crystal in this study have rarely been analyzed by EI and CI mass spectrometry. The infrared spectra of PDAEt with various irradiation times have been studied. Upon irradiation, the most notable spectral changes were the decreases in the absorption bands at $1640 \mathrm{~cm}^{-1}$ and $970 \mathrm{~cm}^{-1}$ assigned to olefinic double bonds. The results indicate that the $[2+2]$ photocyclization reaction occurred between two 


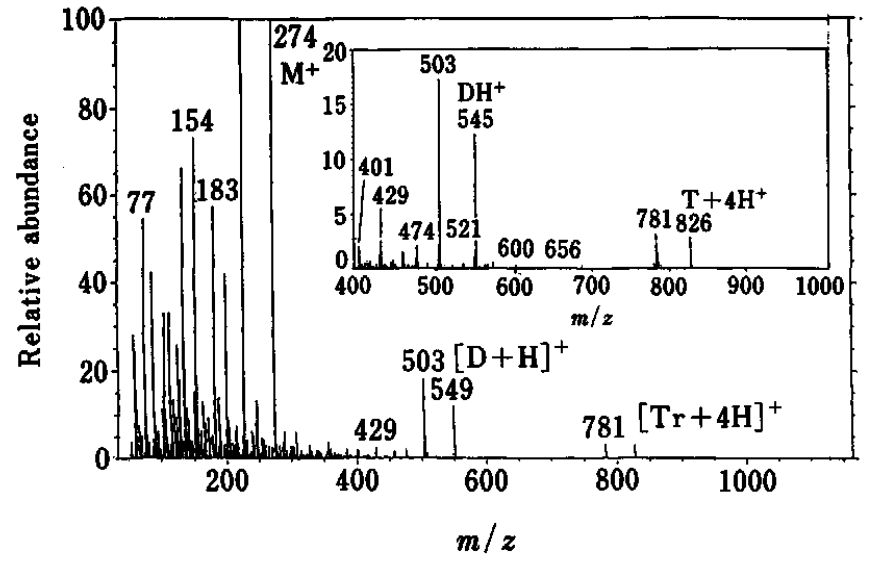

Fig. 1 FAB mass spectrum of oligomers of PDAEt produced by $40 \mathrm{~min}$ photo-irradiation. Matrix, 3-nitrobenzyl alcohol (NBA).

facing double bonds in the crystal. The FAB mass spectrum of PDAEt irradiated for $\mathbf{4 0} \mathrm{min}$ is presented in Fig. 1. As a matrix, 3-nitrobenzyl alcohol (NBA) was better than glycerol for obtaining FAB spectra of these oligomers. FAB spectrum shows many low mass peaks, e.g., at $m / z 77,154,183$ and 226 . The molecular peak of the monomer at $m / z 274$ is very intense. The peaks at $\mathrm{m} / \mathrm{z} 503$ and 777 correspond to the fragment ions due to the elimination of an ethanol molecule from the protonated dimer and trimer, respectively. The peak at $m / z 826$ corresponding to the trimer $+4 \mathrm{H}$ indicated that one double bond in the trimer molecule was reduced probably during the ionization process. We were unsuccessful in analyzing the oligomers larger than tetramer by FAB mass spectrometry.

As described previously, the main purpose of the present study is to determine the effects of irradiation time on the molecular weight distributions of the oligomers by LI mass spectrometry. It is important to select a good matrix in LI method. A good matrix is a proton donor, having a small proton affinity (PA), it can dissolve or disperse analyte molecules at the needle tip, and it reduces the energy required for desorption, resulting in the enhancement of protonated molecule peaks.

Figure 2 shows the LI mass spectra of the oligomers produced by 10 min photo-irradiation of PDAEt crystal. In the case of Fig. 2a), the sample was dissolved in $\mathrm{CHCl}_{3}$ without any matrix. The intense peaks at $m / z 275$ and 549 corresponding to the protonated monomer $\left(\mathrm{MH}^{+}\right)$ and dimer molecules $\left(\mathrm{DH}^{+}\right)$, respectively, were observed, but the peak at $m / z 823$, corresponding to the protonated trimer $\left(\mathrm{TrH}^{+}\right)$was very weak, probably because the solvent $\mathrm{CHCl}_{3}$ vaporized before the desorption of trimer ions. Since hexadecane has been a good matrix for organometallic compounds ${ }^{19}$, hexadecane was used and the oligomers larger than trimer were observed in present work.

In the case of Fig. 2b), the sample mixed with the

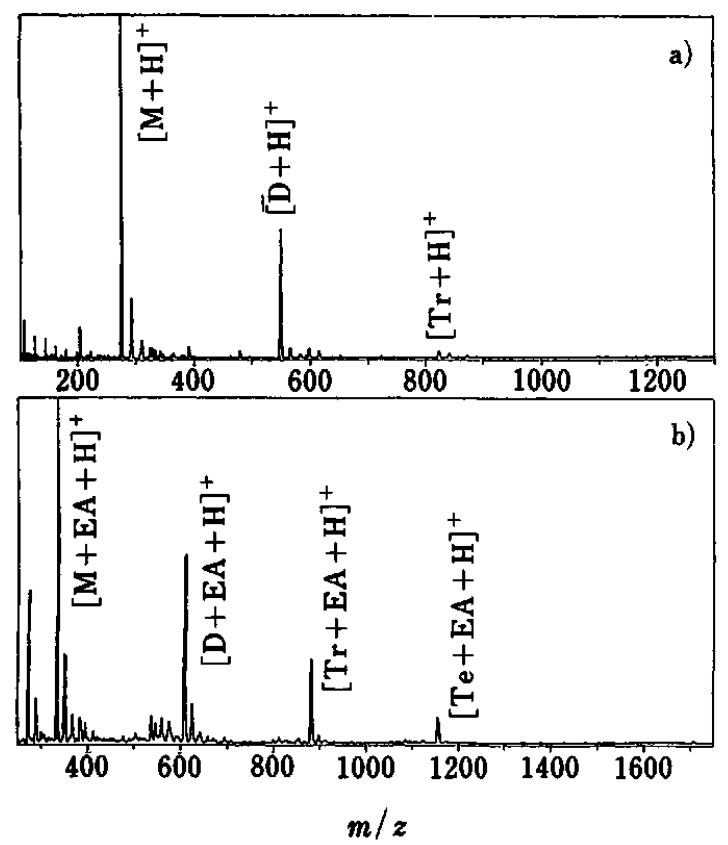

Fig. 2 LI mass spectra of oligomers of PDAEt produced by 10 min photo-irradiation. a): solvent, $\mathrm{CHCl}_{3}$ without any matrix; b): matrix, hexadecane in $\mathrm{CHCl}_{3}(1: 1$ by vol.); reagent, 2-aminoethanol (EA). $[\mathrm{M}+\mathrm{H}]^{+},[\mathrm{D}+\mathrm{H}]^{+},[\mathrm{Tr}+\mathrm{H}]^{+}$, $[\mathrm{Te}+\mathrm{H}]^{+},[\mathrm{Pe}+\mathrm{H}]^{+}$, and $[\mathrm{He}+\mathrm{H}]^{+}$representing as protonated monomer, dimer, trimer, tetramer, pentamer and hexamer, respectively.

matrix solution (hexadecane- $\mathrm{CHCl}_{3}$ solution $1: 1$, by vol.) was deposited on the needle tip. Then 2-aminoethanol, which is used for producing adduct ions, was introduced into the ion source through vapor phase during measurement. The protonated monomer and 2aminoethanol $(E A, M . W .=61)$ adduct ions of monomer, dimer, trimer, and tetramer were observed at $\mathrm{m} / \mathrm{z} 275$, $336,610,884$ and 1158 , respectively. Figure $2 b$ ) shows that almost all oligomer molecules favored to form EA adduct ions and the EA adduct ions of trimer, even tetramer, were observed more abundantly compared with Fig. 2a). Hexadecane (matrix) and 2-aminoethanol (reagent) have good effects to observe larger oligomer ions in these measurements. The results indicate that tetramer and even a traces of hexamer already were produced by $10 \mathrm{~min}$ irradiation, although the monomer was the most abundant.

Products obtained with longer irradiation times, such as 30,40 , and $60 \mathrm{~min}$, were measured using hexadecane and 2-aminoethanol. Figure 3 shows the LI mass spectrum for oligomers produced by $30 \mathrm{~min}$ photoirradiation of PDAEt. Similar to Fig. 2, peaks at $\mathrm{m} / \mathrm{z}$ 275,549 , and 823 corresponding to protonated monomer, dimer, and trimer, respectively, were observed. The EA adduct ions of monomer, dimer, trimer, and tetramer were also observed at $m / z 336,610,884$, and 1158 , respectively. The relative abundances of EA adduct ions were more abundant than those of the 


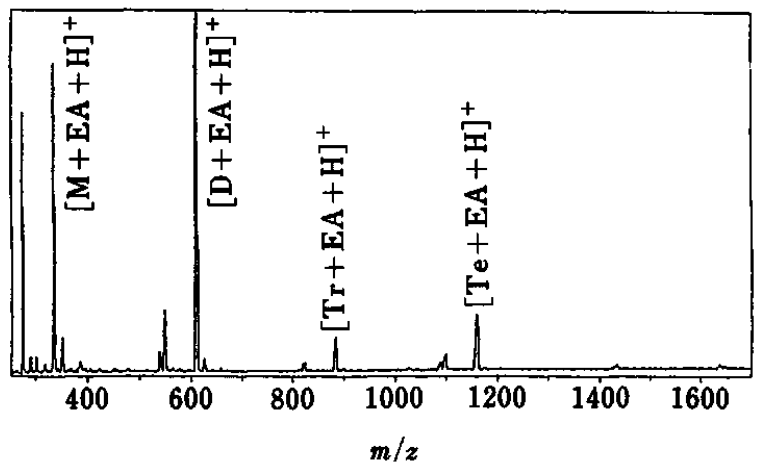

Fig. 3 LI mass spectrum of oligomers of PDAEt produced by 30 min photo-irradiation. Matrix, hexadecane in $\mathrm{CHCl}_{3}$ (1: 1 by vol.); reagent, 2-aminoethanol (EA).
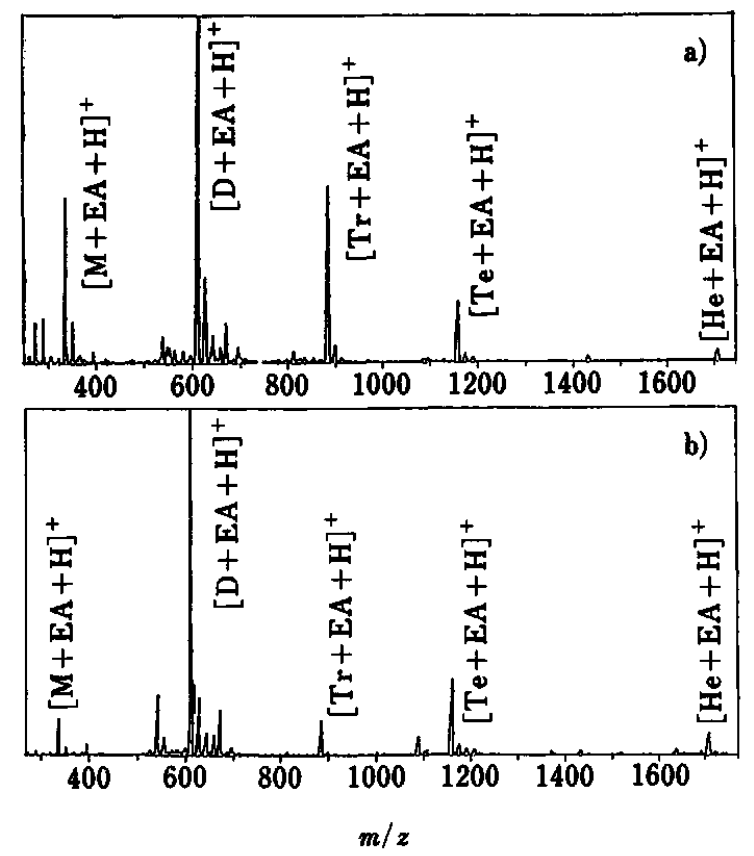

Fig. 4 LI mass spectra of oligomers of PDAEt produced by 40 min photo-irradiation. a) and b) were measured at same conditions: matrix, hexadecane in $\mathrm{CHCl}_{3}$ (1:1 by vol.); reagent, 2-aminoethanol (EA).

corresponding protonated molecules. The mass spectral pattern observed in Fig. 3 indicates that the dimer is the main product produced by $30 \mathrm{~min}$ irradiation.

LI mass spectra (Fig. 4) of the products by $40 \mathrm{~min}$ irradiation show two kinds of product patterns a) and b), which were measured with the same experimental conditions. In both spectra (Fig. 4a) and b)), the EA adduct ions of monomer decreased, the adduct ions of dimer are the most abundant, and those of the tetramer increased with the irradiation time. Some differences, however, were observed in the abundance of trimer ions.

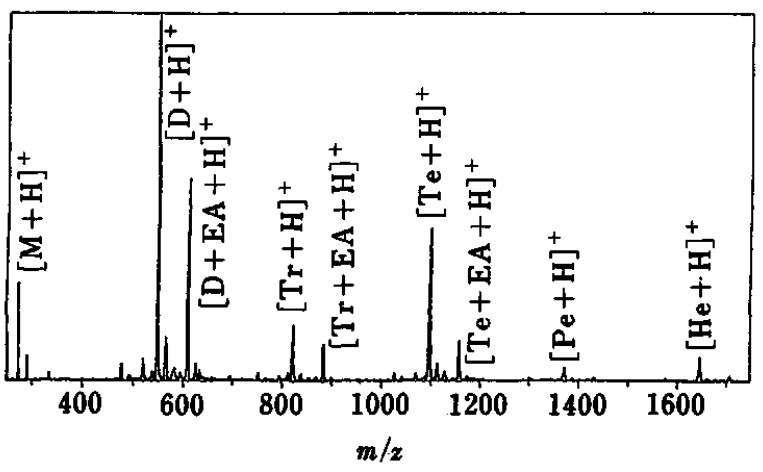

Fig. 5 LI mass spectrum of oligomers of PDAEt produced by $40 \mathrm{~min}$ photo-irradiation. Solvent, trifluoroacetic acid; matrix, hexadecane in $\mathrm{CHCl}_{3}$ (1:1 by vol.); reagent, 2aminoethanol (EA).
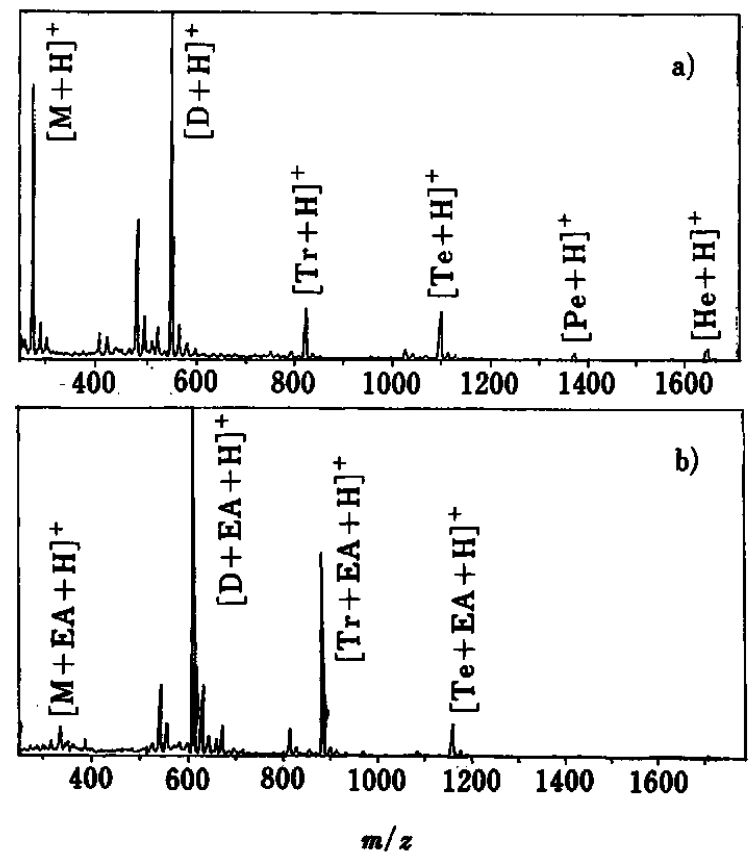

Fig. 6 LI mass spectra of oligomers of PDAEt produced by 60 min photo-irradiation. a): matrix, hexadecane in $\mathrm{CHCl}_{3}$ ( $1: 1$ by vol.); b): matrix, hexadecane in $\mathrm{CHCl}_{3}(1: 1$ by vol.); reagent, 2-aminoethanol (EA).

In the case of a), the trimer adduct ions were more abundant than the tetramer adduct ions. In contrast, in the case of $b$ ), the tetramer adduct ions were more abundant than the trimer adduct ions. A similar trend is observed for pentamer and hexamer, i.e. the hexamer adduct ions were more abundant than the pentamer adduct ions.

Since oligomers larger than trimer were not dissolved in $\mathrm{CHCl}_{3}$, the trifluoroacetic acid was also used as a solvent. All oligomer samples were dissolved in trifluoroacetic acid. Such difference in the sample composition was also observed in the mass spectrum, as 
shown in Fig. 5. In the case of Fig. 5, the sample was dissolved in trifluoroacetic acid and mixed with the same matrix solution (hexadecane $+\mathrm{CHCl}_{3} 1: 1$ ). Two series of peaks corresponding to protonated dimer, trimer, and tetramer as well as their EA adduct ions were observed. This spectrum indicates that the trifluoroacetic acid leads to the formation of protonated oligomer molecules, in spite of existing 2-aminoethanol.

LI mass spectra of PDAEt oligomers produced by photo-irradiation for $60 \mathrm{~min}$ are shown in Fig. 6. From the characteristic peaks for the individual oligomers, the composition of the products can be readily determined. In case of Fig. 6a), oligomer powder was mixed with the matrix solution without addition of 2-aminoethanol. Although the protonated dimer molecule $(\mathrm{m} / \mathrm{z} 549)$ appeared as the base peak, the protonated trimer and tetramer with similar intensities as well as less intense pentamer and hexamer were also observed at $m / z 823$, 1097,1371 and 1647 , respectively. When introducing EA into the LI ion source (Fig. 6b), all oligomer ions observed at $m / z 336,610,884$ and 1158 were corresponding to EA adduct ions of oligomers. The results indicate that the EA adduct ions were more stable than protonated molecules in the case of introduction of EA. The dimer was still the most abundant. In the case of Fig. 6b), the peak intensity of trimer adduct ion increased, compared with Fig. 5 (40 min). In contrast, the peak intensity of monomer adduct ion decreased.

Selected ion profiles, which we call "thermal desorption profiles", are useful to obtain information about the temperature for desorption of the reaction products. Figure 7 shows thermal desorption profiles of the oligomers produced by photo-irradiation for $60 \mathrm{~min}$. The temperature range for desorption of oligomer adduct ions is shown by the thermal desorption profile. As the temperature of the sample increased, monomer ions appeared at about $100^{\circ} \mathrm{C}$, dimer ions at about $140^{\circ} \mathrm{C}$ and trimer ions at about $170^{\circ} \mathrm{C}$. This order is reasonable because of the dependence on their melting points (the m.p. of monomer, dimer, and trimer were $96^{\circ} \mathrm{C}$, $127^{\circ} \mathrm{C}$ and $147^{\circ} \mathrm{C}$ respectively) and molecular size. The tetramer showed two different desorption temperatures: one was higher than that of trimer as expected, but the other one was lower than that of trimer. The latter signal (lower one) may be due to dimeric ions of dimer, $(2 \mathrm{D}+\mathrm{H}+\mathrm{EA})^{+}$.

On photo-irradiation, as was expected, the polymerization proceeded in step-growth mechanism to produce oligomers from dimer, trimer, and larger ones. It is interesting to note that for some samples with longer than 20 min irradiation, tetramer was more abundant than trimer, and hexamer was more abundant than pentamer. For some samples, the trimer was more abundant than the tetramer as expected. As the photo-polymerization were carried out in crystalline state, the structures of oligomers should depend on molecular arrangement of the monomer crystals. Based on step-growth mechanism, the photo-polymerization reaction at the initial stage is considered to yield dimer, trimer and tetramer,

\begin{tabular}{|c|c|}
\hline$m / z 1708$ & \\
\hline \multicolumn{2}{|l|}{1647} \\
\hline \multicolumn{2}{|l|}{1432} \\
\hline \multicolumn{2}{|l|}{1371} \\
\hline 1158 & {$[\mathrm{Te}+\mathrm{EA}+\mathrm{H}]^{+}$} \\
\hline \multicolumn{2}{|l|}{1097} \\
\hline \multicolumn{2}{|l|}{884} \\
\hline \multicolumn{2}{|l|}{823} \\
\hline \multicolumn{2}{|c|}{ 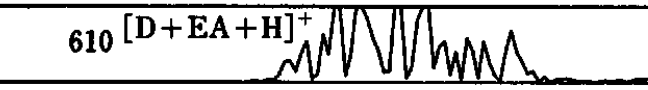 } \\
\hline \multicolumn{2}{|l|}{549} \\
\hline \multicolumn{2}{|l|}{336} \\
\hline \multicolumn{2}{|l|}{275} \\
\hline RIC & \\
\hline
\end{tabular}

Fig. 7 Thermal desorption profiles of oligomers of PDAEt produced by $60 \mathrm{~min}$ photo-irradiation. Matrix, hexadecane in $\mathrm{CHCl}_{3}$ (1:1 by vol.); reagent, 2-aminoethanol (EA).

progressively. When the sample was irradiated for longer than $20 \mathrm{~min}$, reactions between dimer molecules also took place, according to the molecular arrangement. Thermal desorption profiles for oligomers produced by 40 min irradiation (Fig. 8) suggest that the tetramer or hexamer (even numbered oligomers) would be produced from the reactions between dimer and dimer, or dimer and tetramer, because the desorption temperature of dimer and tetramer were lower than those of trimer and pentamer (odd numbered oligomers). Thermal desorption profiles also indicated that the decomposition of oligomer larger than tetramer did not occur in this case (Fig. 8), but did at much higher temperature.

It is interesting to note that the reaction between dimer and dimer observed in present study deviates from a typical step-growth polymerization mechanism. ${ }^{24}$ From the crystal structure of PDAEt ${ }^{25,26}$, equal reaction probability is expected to occur between two intermolecularly facing double bond pairs in the starting crystal, because relative orientation of these double bonds are precisely equivalent. On the other hand, however, after the photoreaction started, the relative orientation of these bonds should deviate gradually from the initial orientation to result in different photoreactivity of each of the residual double bonds in reacting crystals, because during the [2+2] photoreaction, initial orientation should be changed to a certain extent. Such 


\begin{tabular}{|c|c|}
\hline$m / z 1708$ & {$[\mathrm{He}+\mathrm{EA}+\mathrm{H}]^{+}$} \\
\hline \multicolumn{2}{|l|}{1647} \\
\hline 1432 & {$[\mathrm{Pe}+\mathrm{EA}+\mathrm{H}]^{+}$} \\
\hline \multicolumn{2}{|l|}{1371} \\
\hline 1158 & {$[\mathrm{Te}+\mathrm{EA}+\mathrm{H}]^{+}$} \\
\hline \multicolumn{2}{|l|}{1097} \\
\hline 884 & {$[\mathrm{Tr}+\mathrm{EA}+\mathrm{H}]^{+}$} \\
\hline \multicolumn{2}{|l|}{823} \\
\hline \multicolumn{2}{|r|}{$[\mathrm{D}+\mathrm{EA}+\mathrm{H}]^{+}$} \\
\hline \multicolumn{2}{|l|}{549} \\
\hline 336 & {$[\mathrm{M}+\mathrm{EA}+\mathrm{H}]^{+}$} \\
\hline \multicolumn{2}{|l|}{275} \\
\hline$\gamma$ & WUL \\
\hline
\end{tabular}

Fig. 8 Thermal desorption profiles of oligomers of PDAEt produced by $\mathbf{4 0} \mathrm{min}$ photo-irradiation. Matrix, hexadecane in $\mathrm{CHCl}_{3}$ (1:1 by vol.); reagent, 2-aminoethanol (EA).

a photodimerization was observed for the first time in the topochemical behavior of symmetric diolefin compound crystals, although similar behavior has been reported on the unsymmetric monomer crystals in which relative orientation is not equivalent for the double bonds in the starting monomer crystal. 5,6

In conclusion, a suitable matrix, such as hexadecane diluted with $\mathrm{CHCl}_{3}$, is effective to obtain protonated molecular ions. The molecular weight distributions of oligomers up to $\mathrm{m} / z 1644$ are clearly observed with hexadecane and the addition of 2-aminoethanol. The LI technique has advantages over FAB for separation by programmed temperature and for determination of molecular weight of oligomers up to $m / z 2000$ by utilizing suitable matrix and reagents, which provide information about not only the protonated molecules but also molecular adduct ions. The photo-polymerization is mainly by step-growth processes, as reported. ${ }^{3}$ After 20 min photo-irradiation, however, the results indicate that polymerization between dimer molecules also took place, leading to the formation of tetramer and hexamer. These different processes may be attributed to the gradual changes of the molecular arrangement in the initial stage of reaction.

\section{References}

1. M. Hasegawa, Chem. Rev., 83, 507 (1983).

2. M. Hasegawa, "Advances in Physical Organic Chemistry", Vol. 30, p. 117, Academic Press, London, 1995.

3. H. Nakanishi, Y. Suzuki, F. Suzuki and M. Hasegawa, J. Polym. Sci., A-1, 7, 753 (1969).

4. T. Tamaki, Y. Suzuki and M. Hasegawa, Bull. Chem. Soc. Jpn., 45, 1988 (1972).

5. M. Hasegawa, Pure Appl. Chem., 59, 1179 (1986).

6. Y. Maekawa, S. Kato, K. Saigo, M. Hasegawa and Y. Ohashi, Macromolecules, 24, 2314 (1991).

7. T. Tamaki, Y. Suzuki and M. Hasegawa, Bull. Chem. Soc. Jpn., 47, 210 (1974).

8. J. F. Johnson, "Encyclopedia of Polymer Science and Engineering", 2nd ed., Vol. 3, pp. 491 - 531, John Wiley and Sons, New York, 1985.

9. H. R. Schulten and R. P. Lattimer, Mass Spectrom. Rev., 3, 231 (1984).

10. J. Saito, H. Waki, N. Teramae and S. Tanaka, Progr. Org. Coat, 15, 311 (1988).

11. R. P. Lattimer and H. R. Schulten, Int. J. Mass Spectrom. Ion Phys., 67, 227 (1985).

12. C. L. Johlman, C. L. Wilkins, J. D. Hogan, T. L. Donovan, D. A. Laude and M. J. Yousefi, Anal. Chem., 62, $1167(1990)$.

13. S. C. Beu, M. W. Senko, J. P. Quinn, F. M. Wampler and F. W. McLafferty, J. Am. Soc. Mass Spectrom., 4, 557 (1993).

14. F. Hillenkanp, M. Karas, R. C. Beavia and B. T. Chait, Anal. Chem., 63, 1193A (1991).

15. U. Bahr, A. Deppe, M. Karas, F. Hillenkamp and U. Giessmann, Anal. Chem., 64, 2866 (1992).

16. P. O. Danis, D. E. Karr, F. Mayer, A. Holle and C. H. Watson, Org. Mass Spectrom., 27, 843 (1992).

17. M. Tsuchiya, "Advances in Mass Spectrometry", ed. I. Cornides, Gy. Horvath and K. Vekey, Vol. 13, p. 333, John Wiley and Sons, New York, 1995.

18. M. Tsuchiya, J. Mass Spectrom. Soc. Jpn., 43, 373 (1995).

19. M. Tsuchiya and H. Kuwabara, Anal. Chem., 56, 14 (1984).

20. M. Tsuchiya, H. Kuwabara and K. Musha, Anal. Chem., 58, 695 (1986).

21. Y. Niki and M. Tsuchiya, Org. Mass Spectrom., 22, 157 (1987).

22. Y. Yokoyama, Y. Mochida, T. Kawai, S. Nakamura and M. Tsuchiya, J. Mass Spectrom. Soc. Jpn., 44, 483 (1996).

23. Y. Fujioka and M. Tsuchiya, Bunseki Kagaku, 37, 17 (1988).

24. F. W. Billmeyer, "Textbook of Polymer Science", 2nd ed., John Wiley and Sons, New York, 1971.

25. H. Nakanishi, F. Nakanishi, Y. Suzuki and M. Hasegawa, J. Polym. Sci., Polym. Chem. Ed., 11, 2501 (1973).

26. H. Nakanishi, M. Hasegawa and Y. Sasada, J. Polym. Sci., Polym. Phys. Ed., 15, 173 (1977).

(Received November 11, 1996) (Accepted December 18, 1996) 